\title{
Exploiting Redundancy In Iterative H.264 Joint Source and Channel Decoding For Robust Video Transmission
}

\author{
Nasruminallah and L. Hanzo \\ School of ECS, University of Southampton, SO17 1BJ, UK. \\ http: //www-mobile.ecs.soton.ac.uk, \\ Email: $\{1 \mathrm{~h}\} @ e c s$. soton.ac.uk
}

\begin{abstract}
In this paper we propose joint optimisation of soft-bit assisted iterative joint source and channel decoding with the aid of our proposed EXIT chart optimised redundant source mapping (RSM) designed for guaranteed convergence to achieve an infinitesimally low bit error ratio (BER). Data-Partitioned (DP) H.264 source coded video is used to evaluate the performance of our system employing an iterative combination of RSM assisted soft-bit source decoding (SBSD) and recursive systematic convolution codes (RSC) for transmission over correlated narrowband Rayleigh fading channels. EXIT Charts were utilised to analyse the effect of redundancy using different RSM schemes on the attainable system performance, while keeping the overall bitrate budget constant. Explicitly, our experimental results show that the proposed error protection scheme using $\mathbf{R S M}_{2}^{6}$ with $d_{H, \min }=4$ outperform the $\mathbf{R S M}_{2}^{3}$ scheme having $d_{H, \text { min }}=2$ by about $5 d B$, which in turn outperforms the $\mathbf{R S M}_{5}^{6}$ scheme having an identical $d_{H, \text { min }}$ and overall system code-rate by about $2 d B$ at the $P S N R$ degradation point of $2 \mathrm{~dB}$. Additionally, an $E_{b} / N_{0}$ gain of $20 \mathrm{~dB}$ is attained using iterative soft-bit source and channel decoding with the aid of $\operatorname{RSM}_{2}^{3}$ relative to the identical-rate benchmarker.
\end{abstract}

\section{Motivation AND BACKGROUND}

Various multimedia compression standards have been developed to transmit audio and video [1] over band-limited channels. Most of these standards focus on the removal of redundancy from the data stream. However the removal of redundancy makes the data stream more vulnerable to transmission errors. Therefore, the reliable transmission of compressed multimedia source coded streams over diverse wireless communication networks constitutes a challenging research topic [1]. In this scenario the joint optimisation of conventionally separate functions, such as joint source and channel decoding (JSCD), attracted considerable research interests [2]. The family of JSCD schemes often relies on exploiting the residual redundancy in the source-coded bit-stream. Fingscheidt and Vary [3] proposed softbit source decoding (SBSD) in order to exploit the natural residual redundancy of the source-coded bit-stream for Iterative SourceChannel Decoding (ISCD) [4] convergence improvement. However, only modest residual redundancy is left in the source coded bitstream, when using sophisticated state-of-the-art coding techniques, therefore we propose to deliberately impose additional redundancy on the source coded bit-stream with the aid of our novel class of redundant source mapping (RSM) schemes. In our experimental setup the H.264/AVC video codec [5] is used to encode the input video sequence and to generate the source coded bitstream. The H.264/AVC codec employs heterogeneous Variable Length Coding (VLC) and predictive coding techniques to achieve a high compression efficiency, which makes the compressed bit-stream susceptible to transmission errors [1]. A single bit error in the coded stream may result in corruption of numerous future codewords. Likewise, due to predictive coding the effects of channel errors may affect the neighboring video blocks due to error propagation. Therefore the transmission of compressed video over wireless systems presents a challenging task. In [1] various error resilient schemes have been proposed, in order to alleviate these problems, but the price paid is increase in computational complexity and potential reduction of the achievable compression efficiency. Data Partitioning (DP) [5] with intrinsic Error resilient capability has been incorporated in the H.264/AVC codec in order to mitigate the effects of channel errors. In H.264/AVC DP results in three different types of streams, each containing specific sets of coding parameters having different degree of importance. Furthermore, a symbol-based soft-input a posteriori probability (APP) decoder was presented in [6], where the residual redundancy was exploited for improved error protection. On the other hand, a novel irregular variable length coding (IrVLC) scheme designed for nearcapacity joint source and channel coding was proposed in [7]. Instead of the well-known convolutional coded ISCD, an ISCD based on two serial concatenated short block codes was proposed by Clevorn et al. [8]. Furthermore, an optimised bit rate allocation scheme using a rate $r^{\star}=1$ inner channel encoder along with $k=3$ to $k^{\star}=6$ source mapping was proposed in [9], and its performance was evaluated relative to conventional ISCD using a rate $r=\frac{1}{2}$ recursive non-systematic convolutional (RNSC) inner code. In [10] a short block code based redundant index assignment and multi-dimensional mapping was used to artificially introduce redundancy and a single iterative loop was employed. Similarly, Clevorn et al. [11] presented a new design and optimisation guidelines for the ISCD's performance improvement using the concept of redundant index assignment in conjunction with specific generator matrices.

Against this background, in this paper we analyse the performance of our proposed RSM schemes designed for guaranteed convergence in iterative SBSD and channel decoding arrangements, noting that these design principles are applicable to wide-ranging multimedia services, such as voice, audio, handwriting etc. Additionally, instead of modelling the sources with the assumption of a specific source correlation model, we based our system design examples on the simulation of the state-of-the-art H.264/AVC source coded bit-stream. Extrinsic Information Transfer Chart (EXIT) charts were utilised to analyse the effect of variation in the RSM coding rate and $d_{H, \text { min }}$ on the attainable system performance.

The rest of the paper is organised as follows. In Section II we provide an overview of our system model. A portrayal of the RSM coding method along with its EXIT characteristics is provided in Section III. The performance of the proposed system is characterised with the aid of our simulation results in Section IV. Finally we offer our conclusions in Section V.

\section{SYSTEM MODEL}

The schematic of our proposed videophone arrangement used as our design example for quantifying the performance of the proposed RSM schemes is shown in Figure 1. At the transmitter side the video sequence is compressed using the H.264 video codec and the generated video source bit-stream $x_{k}$ is mapped or encoded into the bit-string $x_{m}^{\prime}$ by employing the RSM scheme. Afterwards the output bit-string is interleaved using the bit-interleaver $\Pi$ of Figure 1, yielding the interleaved sequence $\bar{x}_{m}$, which is then encoded by the recursive systematic convolution codes (RSC) code 


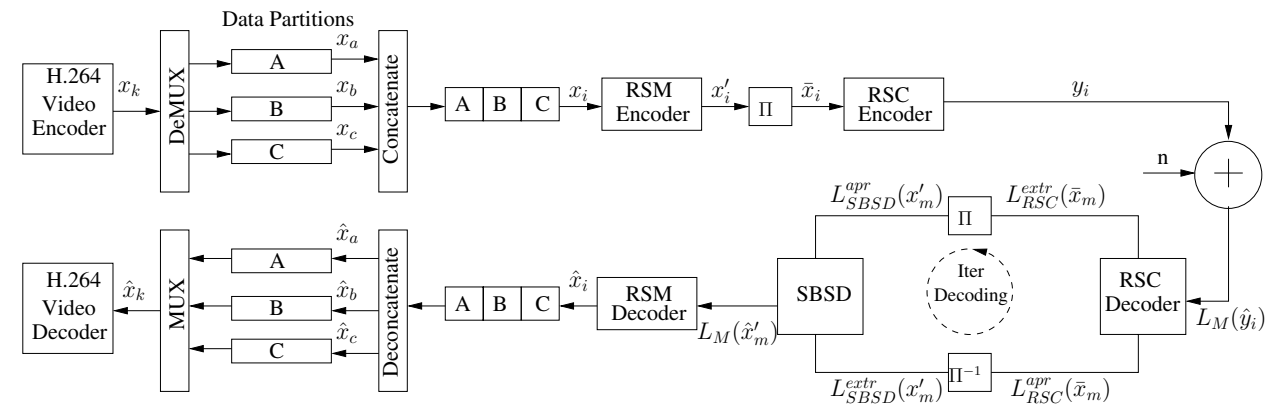

Fig. 1. The proposed system model.

having a specific code rate given in Table III. As the extent of the statistical independence provided by an interleaver is always related to its length [12], instead of performing the ISCD operation on the various frame slices independently, we concatenated all the bits generated by the macro-blocks (MBs) of the slices within a given frame, which results in a longer interleaver without extending the video delay and hence improves the achievable performance of iterative decoding. The resultant bit-stream is QPSK modulated and transmitted over a temporally correlated narrowband Rayleigh fading channel, associated with the normalised Doppler frequency of $f_{d}=f_{D} T_{s}=0.01$, where $f_{D}$ is the Doppler frequency and $T_{s}$ is the symbol duration. At the receiver the signal is QPSK demodulated and the resultant soft-information is transfered to the RSC decoder. The extracted extrinsic information is then exchanged between the SBSD and RSC decoders of Figure 1, in order to attain the lowest possible bit error ratio (BER) [4]. More explicitly, for the employment of RSM scheme the source-encoded bit-stream is partitioned into $M=2^{K}$-ary, or $K$-bit symbols, and will be termed as the information word to be encoded by the proposed RSM scheme, each with a different probability of occurance. The redundancy of the source bit-stream is then characterised with the aid of the nonuniform $M=2^{K}$-ary symbol probability distribution $P\left[S_{K}(\tau)\right]$, where $S_{K}(\tau)=\left[S_{K}(1), S_{K}(2), \cdots S_{K}(M)\right]$, with $K$ denoting the number of bits in each $M=2^{K}$-ary symbol.The details regarding extrinsic information generation algorithm using SBSD for the zeroorder Markov model can be obtained from [4].

$$
P\left[\hat{x}_{\tau} \mid x_{\tau}\right]=\prod_{k=1}^{K} P\left[\hat{x}_{\tau}(k) \mid x_{\tau}(k)\right],
$$

where $\hat{x}_{\tau}$ and $x_{\tau}$ are the corresponding transmitted and received $K$ bit source sequences respectively. For each desired bit $\left[x_{\tau}(\lambda)\right]$, the extrinsic channel output information $P\left[\hat{x}_{\tau}^{[e x t]} \mid x_{\tau}^{[e x t]}\right]$ is expressed as:

$$
P\left[\hat{x}_{\tau}^{[e x t]} \mid x_{\tau}^{[e x t]}\right]=\prod_{k=1, k \neq \lambda}^{K} P\left[\hat{x}_{\tau}(k) \mid x_{\tau}(k)\right] .
$$

Finally, the resultant extrinsic Log Likelihood Ratio (LLR) value can be acquired for each bit of the $\tau$-th symbol by combining its channel output information and the a priori knowledge of the corresponding $\tau$-th symbol as [3,4]:

$$
\begin{gathered}
\operatorname{LLR}\left[x_{\tau}(\lambda)\right]= \\
\log \left(\frac{\sum_{x_{\tau}^{[e x t]}} P\left[x_{\tau}^{[e x t]} \mid x_{\tau}(\lambda)=+1\right] \cdot P\left[\hat{x}_{\tau}^{[e x t]} \mid x_{\tau}^{[e x t]}\right]}{\sum_{x_{\tau}^{[e x t]}} P\left[x_{\tau}^{[e x t]} \mid x_{\tau}(\lambda)=-1\right] \cdot P\left[\hat{x}_{\tau}^{[e x t]} \mid x_{\tau}^{[e x t]}\right]}\right) .
\end{gathered}
$$

In our design example the source coded bit-stream's redundancy is characterised with the aid of the non-uniform $M$-ary symbol probability distribution using the H.264/AVC video encoded bit-stream of the 300 frame "Akiyo" video sequence, the 300-frame "Mother\&Daughter" video clip and the 150 frame
"MissAmerica" video sequence which were used as training sequences.

\section{Redundant Source Mapping Assisted Iterative SOURCE CHANNEL DECODING}

The intention of ISCD is to utilise the constituent inner and outer decoders in order to assist each other in an iterative fashion to glean the highest possible extrinsic information from each other. However, the achievable performance improvements of SBSD may remain limited due to the limited residual redundancy in the video-encoded bit-stream, when using the H.264/AVC video codec with highcompression efficiency as depicted in Figure 2. It may be observed from the simulation results of [13] that typically using SBSD results in negligible system performance improvements beyond two decoding iterations. Hence, we artificially introduce redundancy in the source coded bit-stream using our proposed RSM coding, in order to improve the achievable ISCD performance gain. The novel philosophy of our RSM design is based on exploiting a specific property of EXIT Charts [14]. More explicitly, the sufficient and necessary condition for the near-capacity operation of iterative detection was shown by Kliewer [15] to be that the legitimate codewords should have $d_{H, \min }=2$. Then the ISCD scheme becomes capable of achieving the highest possible source entropy denoted as $H(X)=L_{S B S D}^{e x t r}=$ $1 \mathrm{bit}$, provided that the input a priori information of the SBSD is perfect, i.e. we have $H(X)=L_{S B S D}^{a p r i}=1$ bit. This motivates the design of our novel RSM schemes referred to as Mapping- $I$ and $I I$, which maps or encodes each $K$-bit symbol of the source set $X$ to the $N$-bit code words of the RSM set $f(X)$, while providing $d_{H, \min } \geq 2$.

Mapping- $I$ :

According to our $\mathrm{RSM}_{K}^{N}$ Mapping- $I$ encoding procedure, the $K$-bit information word is encoded into $N=(K+1)$-bits consisting of information bits and an additional redundant bit $r_{\tau}$. The redundant bit $r_{\tau}$ is generated for the $\tau$-th $M$-ary source symbol by calculating the exclusive $O R(X O R)$ function of its $K$ constituent bits, as follows:

$$
r_{\tau}=\left[b^{\tau}(1) \oplus b^{\tau}(2) \ldots \oplus b^{\tau}(K)\right],
$$

where $\oplus$ represents the $X O R$ operation.

For a specific $K$ to $N$-bit RSM coding, any one of the $(K+1)$ different bit positions can be selected to incorporate the resultant redundant bit of a source symbol, in order to create $(K+1)$ different RSM combinations, each having $d_{H, \min }=2$, as shown in Table I for a case of incorporating the redundant bit $r_{\tau}$ at the end of the $\tau_{t h}$ $K$-bit source symbol.

Mapping- $I I$ :

In order to further decrease the RSM coding rate of Mapping-I and to increase its $d_{H, \text { min }}$, we introduce Mapping- $I I$ in which the $N$ additional bits are concatenated to the bits encoded according to Mapping- $I$ by repeating the same coded bits in a reverse order, which results in a $K$ to $(2 \times N)$-bit mapping, where we have $N=$ $(K+1)$, as depicted in Table I.

Let us now demonstrate the power of RSM with the aid of a design example. As an example, the various RSM mapping symbols 
TABLE I

$[\mathrm{N}+1]$ DIFFERENT RSM COMBINATIONS.

\begin{tabular}{|c|c|c|}
\hline Input Symbols & Mapping-I Symbols & Mapping-II Symbols \\
\hline$S_{(1)}$ & $r_{1} b_{1} b_{2} . b_{K}$ & $r_{1} b_{1} b_{2} \cdots b_{K} b_{K} \cdots b_{2} b_{1} r_{1}$ \\
$S_{(2)}$ & $r_{2} b_{1} b_{2} . . b_{K}$ & $r_{2} b_{1} b_{2} \cdots b_{K} b_{K} \cdots b_{2} b_{1} r_{2}$ \\
$\vdots$ & $\vdots$ & $\vdots$ \\
$\left.S_{(2}{ }\right)$ & $r_{2 K} b_{1} b_{2} . b_{K}$ & $r_{2} b_{1} b_{2} \cdots b_{K} b_{K} \cdots b_{2} b_{1} r_{2}$ \\
\hline
\end{tabular}

generated by applying the proposed $\mathrm{RSM}_{K}^{N}$ encoding schemes along with their corresponding $d_{H, \text { min }}$ is summarised in Table II. Again, as it becomes evident from Table II, the EXIT-chart optimised RSM ensure that the mapped symbols exhibit $d_{H, \min } \geq 2$. Additionally, only $2^{K}$ out of either the $2^{N}$ possible $N$-bit symbols of Mapping$I$ or $2^{(N \times 2)}$ possible $(N \times 2)$-bit symbols of Mapping-II are legitimate in the mapped source coded bit-stream, where $N=(K+$ 1 ), which exhibits a non-uniform probability of occurance for the $N$ bit mapped source symbols. Figure 2 portray the EXIT characteristics of the SBSD scheme of Figure 1 using either the rate- $1 \mathrm{RSM}^{\star 3}$ or the rate $<1$ RSM schemes shown in Table II. More specifically, the EXIT curve of SBSD using rate $<1$ RSM schemes does indeed reach to the top right corner of the EXIT chart at $\left(I_{A}, I_{E}\right)=(1,1)$ and hence results in an infinitesimally low BER. By contrast, the SBSD scheme using a rate- $1 \mathrm{RSM}^{\star}$, i.e. no RSM fails to do so. TABLE II

DIFFERENT RSM SCHEMES WITH CORRESPONDING SYMBOLS AND $d_{H, \min }$

\begin{tabular}{|l|l|c|}
\hline RSM Type & Symbols in Decimal & {$\left[d_{H, \text { min }}\right]$} \\
\hline \hline Rate1 RSM & $\{0,1\}$ & 1 \\
Rate- $\frac{2}{3} \mathrm{RSM}_{2}^{3}$ & $\{0,3,5,6\}$ & 2 \\
Rate- $\frac{3}{4} \mathrm{RSM}_{3}^{4}$ & $\{0,3,5,6,9,10,12,15\}$ & 2 \\
Rate- $\frac{4}{5} \mathrm{RSM}_{4}^{5}$ & $\{0,3,5,6,9,10,12,15,17,18,20,23,24,27,29,30\}$ & 2 \\
Rate- $\frac{5}{6} \mathrm{RSM}_{5}^{6}$ & $\{0,3,5,6,9,10,12,15,17,18,20,23,24,27,29,30,33$, & 2 \\
& $34,36,39,40,43,45,46,48,51,53,54,57,58,60,63\}$ & \\
\hline Rate- $\frac{1}{3} \mathrm{RSM}_{2}^{6}$ & $\{0,30,45,51\}$ & 4 \\
Rate- $\frac{3}{8} \mathrm{RSM}_{3}^{8}$ & $\{0,60,90,102,153,165,195,255\}$ & 4 \\
Rate- $\frac{2}{5} \mathrm{RSM}_{4}^{10}$ & $\{0,120,180,204,306,330,390,510,561,585,645$, & 4 \\
& $765,771,891,951,975\}$ & 4 \\
Rate- $\frac{5}{12} \mathrm{RSM}_{5}^{12}$ & $\{0,240,360,408,612,660,780,1020,1122,1170$, & \\
& $1290,1530,1542,1782,1902,1950,2145,2193$, & \\
& $2313,2553,2565,2805,2925,2973,3075,3315\}$, & \\
& $3435,3483,3687,3735,3855,4095\}$ & \\
\hline
\end{tabular}

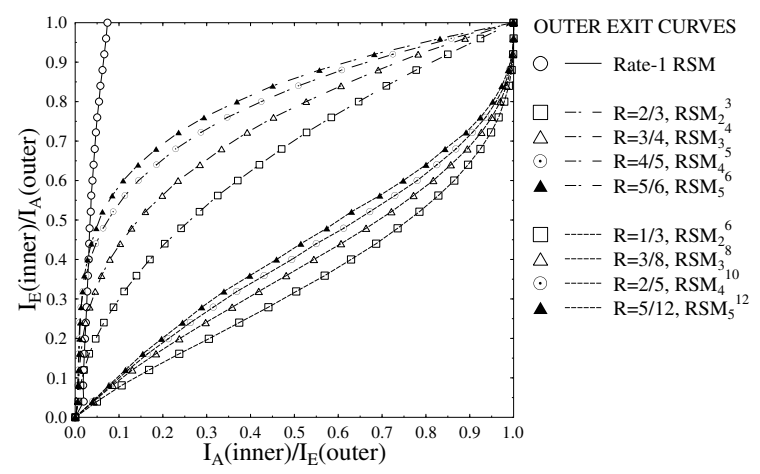

Fig. 2. EXIT characteristics of SBSD with the aid of various rate RSM.

The coding parameters of the different RSM schemes used in our

${ }^{3}$ For the sake of using a unified terminology, we refer to the scheme using no RSM as the rate- 1 RSM $^{\star}$. design example are shown in Table III. We considered a concatenated rate $R=\frac{1}{4}$ RSC encoder having a code memory of 4 and octally represented generator polynomials of $\left(G_{1}, G_{2}, G_{3}, G_{4}\right)=$ $(13,15,15,17)_{8}$. Observe from the Table III that an overall coderate of $R=\frac{1}{4}$ was maintained by adjusting the puncturing rate of the concatenated RSC in order to accommodate the different RSM rates of Table II, while keeping the overall bit-rate budget constant.

TABLE III

CODE RATES FOR DIFFERENT ERROR PROTECTION SCHEMES

\begin{tabular}{|c|c|c|c|}
\hline \multirow{2}{*}{ Error Protection Scheme } & \multicolumn{3}{|c|}{ Code Rate } \\
\hline & RSC & RSM & Overall \\
\hline rate-1 $\mathrm{RSM}^{\star}$ & $1 / 4$ & $\overline{1}$ & $\overline{1 / 4}$ \\
\hline $\mathrm{RSM}_{2}^{3}$ & $3 / 8$ & $2 / 3$ & $1 / 4$ \\
\hline $\mathrm{RSM}_{3}^{4}$ & $1 / 3$ & $3 / 4$ & $1 / 4$ \\
\hline $\mathrm{RSM}_{4}^{5}$ & $5 / 16$ & $4 / 5$ & $1 / 4$ \\
\hline $\mathrm{RSM}_{5}^{6}$ & $3 / 10$ & $5 / 6$ & $1 / 4$ \\
\hline $\mathrm{RSM}_{2}^{6}$ & $3 / 4$ & $2 / 6$ & $1 / 4$ \\
\hline $\mathrm{RSM}_{3}^{8}$ & $2 / 3$ & $3 / 8$ & $1 / 4$ \\
\hline $\mathrm{RSM}_{4}^{10}$ & $5 / 8$ & $4 / 10$ & $1 / 4$ \\
\hline $\operatorname{RSM}_{5}^{12}$ & $3 / 5$ & $5 / 12$ & $1 / 4$ \\
\hline
\end{tabular}

\section{System Performance Results}

In this section we present the performance results of our proposed system. We used a 45 frame "Akiyo" video sequence [1] in (176x144)-pixel Quarter Common Intermediate Format (QCIF) as our test sequence. This test sequence was encoded using the H.264/AVC JM 13.2 reference video codec at 15 frames-per-second $(f p s)$ at the target bitrate of $64 \mathrm{kbps}$. Each QCIF frame was partitioned into 9 slices and each slice was composed of $11 \mathrm{MBs}$. The resultant video encoded clip comply to the intra-coded 'I' and predicted 'P' frame sequence, consisting of an 'I' frame followed by 44 ' $\mathrm{P}$ ' frames, corresponding to 3 seconds lag between two consecutive 'I' frames at $15 \mathrm{fps}$, in order to reduce error propagation. Additionally, we incorporated error resilience features, such as DP and intra-frame coded MB updates of three randomly distributed MBs per frame, to control the effects of error propagation. The insertion of ' $B$ ' pictures results in an unacceptable loss of lip-synchronisation as a result of the corresponding delay incurred due to the bi-directionally predicted video coding operations [5], and hence was avoided. Keeping in view the videophone senario, error resilient encoding techniques, such as Flexible Macro-block Ordering (FMO) [5] and the employment of multiple reference frames for inter-frame motion compensation were turned off, because despite their substantially increased complexity they typically result in modest video performance improvements in low-motion head-and-shoulders video sequences, such as the "Akiyo" clip. Additionally, only the immediately preceding frame was used for motion search, which results in a reduced computational complexity compared to using multiple reference frames. Moreover, due to the limited residual redundancy inherent in the source encoded bit-stream and for the sake of reducing the computational complexity imposed, we limited the number of iterations between the RSC and SBSD decoders to $I_{t}=5$, when using a rate-1 RSM - i.e. no RSM. By contrast we used $I_{t}=10$ iterations, when applying RSM schemes having a rate below unity. For the sake of increasing the confidence in our results, we repeated each 45 -frame experiment 160 times and averaged the generated results. Addtitionally, the performance of our proposed system was evaluated by keeping the same overall code rate as well as video rate for the different considered error protection schemes. 
The actual decoding trajectories of the various error protection schemes employing the different Mapping-I RSM schemes along with their corresponding Mapping-II RSM schemes as well as using the respective constituent inner RSCs detailed in Table III was recorded at $E_{b} / N_{0}=-0 d B,-1 d B$ and $E_{b} / N_{0}=-3.0 d B$, $-3.5 d B$ respectively, as portrayed in Figures $3,4,5$, and 6 . These trajectories were recorded by acquiring the mutual information at the input and output of both the inner and outer decoder during the bit-bybit Monte-Carlo simulation of the iterative soft-bit source and channel decoding algorithm. It may be inferred from the EXIT trajectories of Figures 3, 4, 5, and 6 that as expected, the convergence behaviour of the Mapping- $I$ RSM coding improves upon decreasing the RSM coding rate, which can further be improve upon the employment of the corresponding Mapping-II RSM coding, with additional redundancy and improved $d_{H, m i n}$.

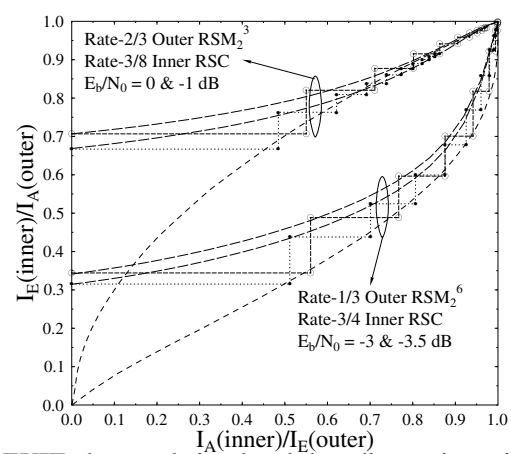

Fig. 3. The EXIT chart and simulated decoding trajectories using the $\mathrm{RSM}_{2}^{3}$ and $\mathrm{RSM}_{2}^{6}$ schemes of Table III.

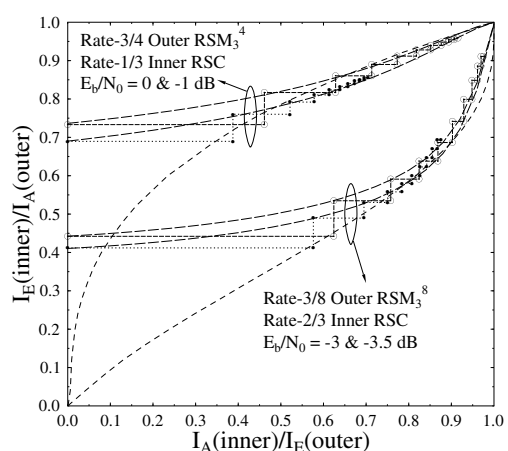

Fig. 4. The EXIT chart and simulated decoding trajectories of the $\mathrm{RSM}_{3}^{4}$ and $\mathrm{RSM}_{3}^{8}$ schemes of Table III.

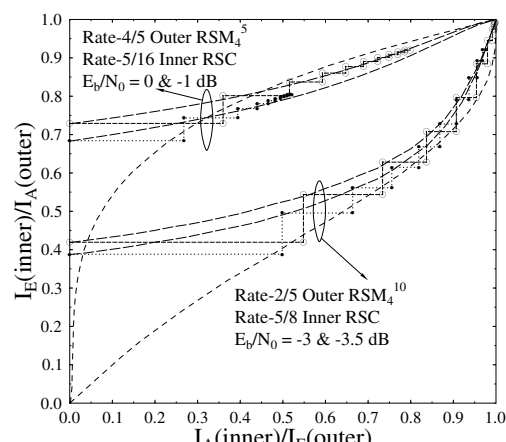

Fig. 5. The EXIT chart and simulated decoding trajectories of the $\mathrm{RSM}_{4}^{5}$ and $\mathrm{RSM}_{4}^{10}$ schemes of Table III.

Figure 7 presents the performance of the various rate RSM based error protection schemes of Table II in terms of the attainable BER along with the AWGN optimum performance curve, while their comparison with the rate- $1 \mathrm{RSM}^{\star}$ based schemes is offered in Figures 9. The performance trends expressed in terms of the $P S N R$

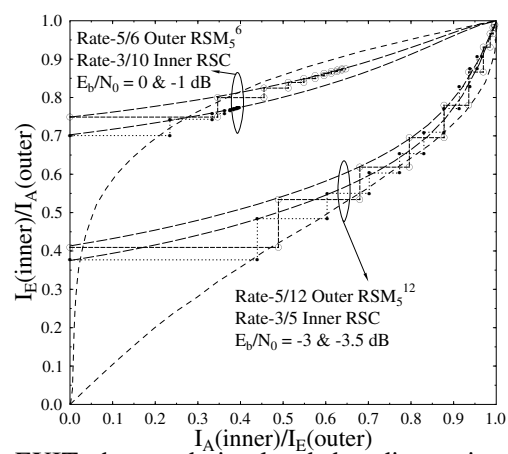

Fig. 6. The EXIT chart and simulated decoding trajectories of the RSM 6 and $\mathrm{RSM}_{5}^{12}$ schemes of Table III

versus $E_{b} / N_{0}$ curves are portrayed in Figures 8 and 10 along with the reference AWGN performance curves. It may be observed in Figure 8 that the $\mathrm{RSM}_{2}^{6}$ scheme with lowest coding rate among the different considered RSM schemes of Table III provides the best $P S N R$ performance across the entire $E_{b} / N_{0}$ region considered. Its also observed in Figure 10 that when using SBSD in conjunction with the rate- 1 outer $\mathrm{RSM}^{\star}$ and rate- $\frac{1}{4}$ inner RSC results in a worse $P S N R$ performance than the RSM schemes having a rate below unity combined with their respective inner RSCs, at the same overall code rate of $\frac{1}{4}$, as mentioned in Table III. Quantitatively, using the RSM of Table III having a rate lower than 1 , an additional $E_{b} / N_{0}$ gain of upto $20 \mathrm{~dB}$ may be achieved over the rate-1 RSM ${ }^{\star}$.

Finally, the subjective video quality achieved by the proposed error protection schemes consisting of Mapping-I RSM coding was recorded in Figure 11 at the channel $E_{b} / N_{0}$ value of $0.5 \mathrm{~dB}$. The corresponding results achieved by Mapping-II RSM coding at $E_{b} / N_{0}$ value of $-3.0 d B$ were presented in Figure 12. In order to have a fair subjective video quality comparison, we present both the average and cumulative-error results of both the luminance and chrominance components of the 30 "Akiyo" video test sequences described in Section IV, decoded using the H.264 video codec after transmission through our proposed system for each type of setup. Observe from Figure 11 that the achievable video quality improves upon decreasing the Mapping-I RSM code rate. Additionally, its clear from Figure 12 that the employment of the Mapping-II RSM scheme provides an improved video quality at $3.5 d B$ lower $E_{b} / N_{0}$ value raletive to the results of various Mapping-I RSM schemes presented in Figure 11

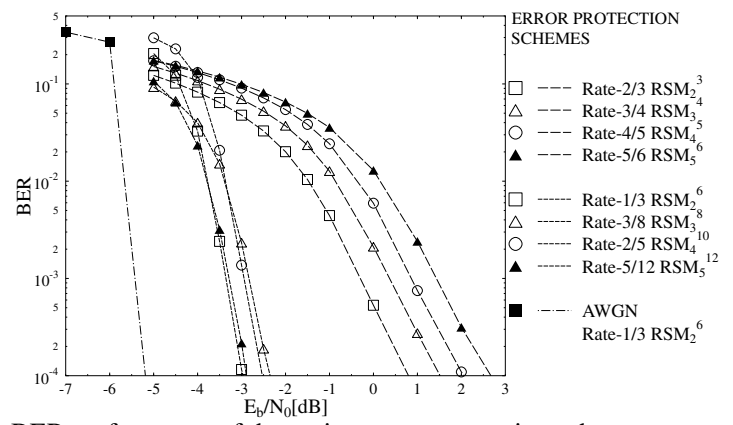

Fig. 7. BER performance of the various error protection schemes summarised in Table III.

\section{Conclusions}

In this paper we analysed the effects of artificial redundancy using a generic low-complexity RSM coding scheme on the performance of arbitrary SBSD-aided multimedia source codecs. We applied diverse error protection schemes considering the transmission of DP aided H.264/AVC coded video using carefully selected RSM 


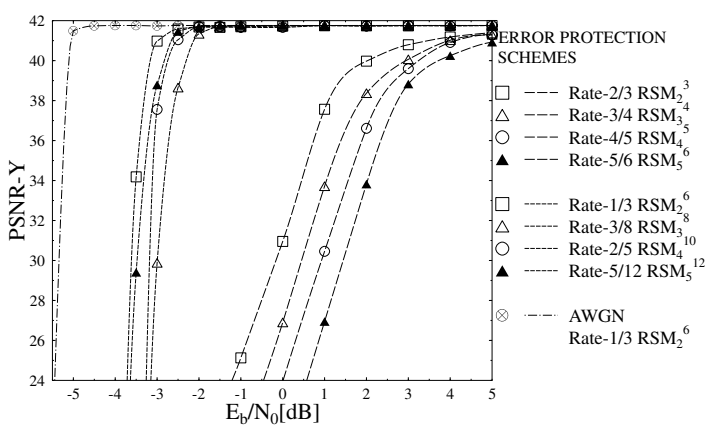

Fig. 8. PSNR-Y performance of the various error protection schemes summarised in Table III.

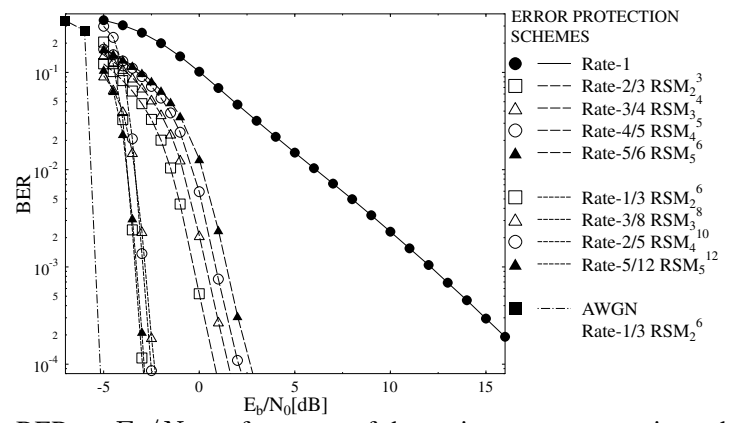

Fig. 9. BER vs $E_{b} / N_{0}$ performance of the various error protection schemes summarised in Table III.

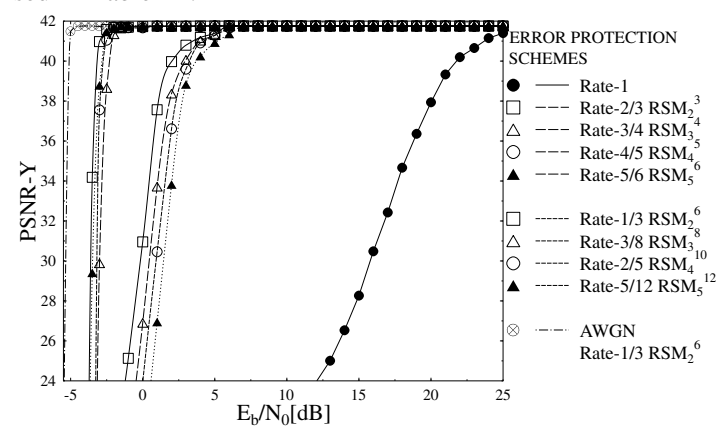

Fig. 10. PSNR-Y vs $E_{b} / N_{0}$ performance of various error protection schemes summarised in Table III.

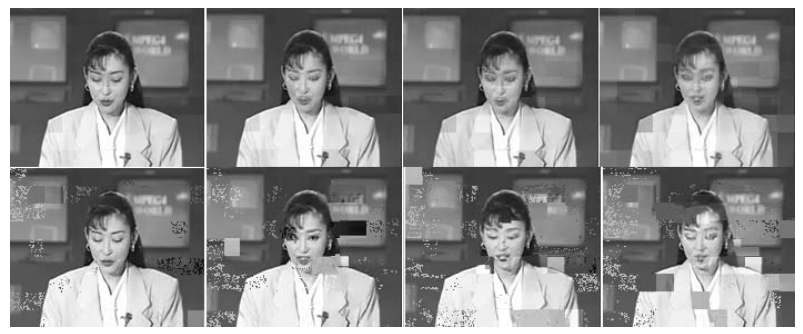

Fig. 11. Subjective video quality of the $45^{\text {th }}$ "Akiyo" video sequence frame in terms of (from top) average and cumulative-error video quality using (from left) Rate- $\frac{2}{3}, \frac{3}{4}, \frac{4}{5}$ and $\frac{5}{6}$ Mapping-I RSM summarised in Table III at $E_{b} / N_{0}=0.5 \mathrm{~dB}$

schemes having different coding-rates and hence redundancy. It was demonstrated that the bit-error correction capability of the ISCD scheme was significantly improved with the advent of rate $<1$ RSM scheme owing to the deliberate increase in redundancy of the source coded bit-stream, when we beneficially partitioned the total available bit rate budget between the source and channel codecs. Additionally, EXIT charts were used to analyse the convergence behaviour of the ISCD system. Our design based on the H.264, RSM and RSC constituent components exhibit an $E_{b} / N_{0}$ gain of $5 \mathrm{~dB}$ at the $P S N R$ degradation point of $2 d B$ when using $\mathrm{RSM}_{2}^{6}$ with $d_{H, \min }=4$

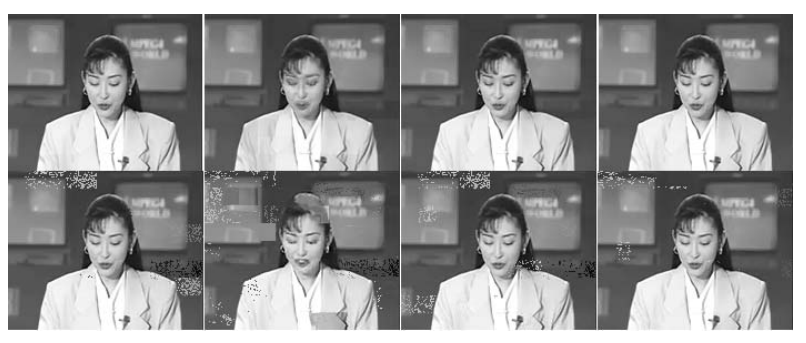

Fig. 12. Subjective video quality of the $45^{\text {th }}$ "Akiyo" video sequence frame in terms of (from top) average and cumulative-error video quality using (from left) Rate- $\frac{2}{6}, \frac{3}{8}, \frac{4}{10}$ and $\frac{5}{12}$ Mapping-II RSM summarised in Table III at $E_{b} / N_{0}=-3.0 \mathrm{~dB}$.

compare to the employment of $\mathrm{RSM}_{2}^{3}$ having $d_{H, \text { min }}=2$, which in turn outperforms the $\mathrm{RSM}_{5}^{6}$ having an identical $d_{H, \text { min }}$ and overall system code-rate by about $2 d B$. Moreover, an $E_{b} / N_{0}$ gain of $20 d B$ was attained with the aid of the $\mathrm{RSM}_{2}^{3}$ relative to the identical-rate benchmarker dispensing with RSM.

\section{REFERENCES}

[1] L. Hanzo, P. Cherriman, and J. Streit, Video Compression and Communications: From Basics to H.261, H.263, H.264, MPEG2, MPEG4 for DVB and HSDPA-Style Adaptive Turbo-Transceivers. Wiley-IEEE Press, 2007.

[2] Nasruminallah and L. Hanzo, "Exit-chart optimised short block codes for iterative joint source and channel decoding in h.264 video telephony," IEEE Transactions on Vehicular Technology, 2009.

[3] T. Fingscheidt and P. Vary, "Softbit speech decoding: a new approach to error concealment," IEEE Transactions on Speech and Audio Processing, vol. 9, pp. 240-251, Mar. 2001.

[4] M. Adrat and P. Vary, "Iterative source-channel decoding: improved system design using EXIT charts," EURASIP J. Appl. Signal Process., vol. 2005, no. 1, pp. 928-941, 2005.

[5] T. Stockhammer, M. M. Hannuksela, and T. Wiegand, "H.264/AVC in wireless environments," IEEE Transactions on Circuits and Systems for Video Technology, vol. 13, pp. 657-673, July 2003.

[6] J. Kliewer and R. Thobaben, "Iterative joint source-channel decoding of variable-length codes using residual source redundancy," IEEE Transactions on Wireless Communications, vol. 4, pp. 919-929, May 2005.

[7] R. G. Maunder, J. Wang, S. X. Ng, L. L. Yang, and L. Hanzo, "On the performance and complexity of irregular variable length codes for near-capacity joint source and channel coding," IEEE Transactions on Wireless Communications, vol. 7, pp. 1338-1347, Apr. 2008.

[8] T. Clevorn, P. Vary, and M. Adrat, "Iterative Source-channel Decoding Using Short Block Codes," in Acoustics, Speech and Signal Processing, 2006. ICASSP 2006 Proceedings. 2006 IEEE International Conference on, vol. 4, May 2006.

[9] M. Adrat, P. Vary, and T. Clevorn, "Optimized bit rate allocation for iterative source-channel decoding and its extension towards multimode transmission," in in Proceedings of IST Mobile and Wireless Communications Summit (Dresden, Germany), pp. 1153-1157, June 2005.

[10] T. Clevorn, M. Adrat, and P. Vary, "Turbo decodulation using highly redundant index assignments and multi-dimensional mappings," Proceedings of International Symposium on Turbo Codes \& Related Topics, Apr. 2006.

[11] T. Clevorn, L. Schmalen, P. Vary, and M. Adrat, "On Redundant Index Assignments for Iterative Source-channel Decoding," IEEE Communications Letters, vol. 12, pp. 514-516, July 2008.

[12] R. G. Maunder, J. Kliewer, S. X. Ng, J. Wang, L. L. Yang, and L. Hanzo, "Joint iterative decoding of trellis-based VQ and TCM," IEEE Transactions on Wireless Communications, vol. 6, pp. 1327-1336, Apr. 2007.

[13] T. Hindelang, M. Adrat, T. Fingscheidt, and S. Heinen, "Joint source and channel coding: from the beginning until the 'EXIT'," European Transactions on Telecommunications, vol. 18, no. 8, pp. 851-858, 2007.

[14] S. ten Brink, "Designing iterative decoding schemes with the extrinsic information transfer chart," in AEU International Journal of Electronics and Communications, vol. 54, pp. 389-398, Nov. 2000.

[15] J. Kliewer, N. Goertz, and A. Mertins, "Iterative source-channel decoding with markov random field source models," IEEE Transactions on Signal Processing, [see also IEEE Transactions on Acoustics, Speech, and Signal Processing,], vol. 54, no. 10, pp. 3688-3701, Oct. 2006. 